\title{
Fauna of Protected Areas - 23: INSECT FAUNA OF PEECHI-VAZHANI WILDLIFE SANCTUARY, KERALA, INDIA
}

\author{
George Mathew ${ }^{1,2}$, R.S.M. Shamsudeen ${ }^{1}$ and Rashmi Chandran ${ }^{1}$ \\ ${ }^{1}$ Division of Forest Protection, Kerala Forest Research Institute, Peechi, Kerala 680653, India \\ Email: ${ }^{2}$ mathew@kfri.org
}

\begin{abstract}
In a study on the insect fauna of Peechi-Vazhani Wildlife Sanctuary, 374 species of insects mostly belonging to Lepidoptera, Coleoptera and Hemiptera were recorded. The fauna was rich and diverse and contained several rare and protected species. Among butterflies, of the 74 species recorded, six species (Chilasa clytia, Appias lyncida, Appias libythea, Mycalesis anaxias, Hypolimnas misippus and Castalius rosimon) are protected under the Indian Wildlife (Protection) Act. Similarly, four species of butterflies, Papilio buddha, Papilio polymnestor, Troides minos, and Cirrochroa thais, recorded in this study are rare and restricted in distribution. The moth fauna is rich in arboreal feeding forms indicating an undisturbed forest patch in the area. Certain species associated with herbaceous ground vegetation are of economic importance, being already reported as pests of various agricultural crops. Beetles are also abundant, being dominated by phytophagous and scavenger forms, the former feeding mostly on herbaceous ground flora and the latter associated with animal excreta. The bugs recorded in this study included mostly phytophagous forms. The hymenopterans contained several species of solitary bees and wasps. An inventory of 382 species of insects recorded from the Sanctuary is given.

\section{KEYWORDS}

Endemic, insect fauna, inventory, Kerala, Peechi-Vazhani Wildlife Sanctuary, protected, rare
\end{abstract}

Peechi-Vazhani Wildlife Sanctuary (P-VWS) situated in Thrissur district, Kerala state $\left(76^{0} 15^{\prime}-76^{0} 27^{\prime} \mathrm{E} \& 10^{0} 30^{\prime}-10^{\circ} 42^{\prime} \mathrm{N}\right)$, is about $125 \mathrm{~km}^{2}$ in extent (Fig. 1). It is bordered by the Chimmoni Wildlife Sanctuary on the east and the forests of Palakkad division in the north. The terrain is undulating and the altitude varies from 100 to $914 \mathrm{~m}$ above mean sea level. The reservoir of Peechi dam is located in this area. The climate of the area is generally of a warm humid type. The average annual rainfall ranges between 2000-4000mm with southwest monsoon rains during JuneSeptember contributing a major portion of the precipitation. The relative humidity is usually higher than $55 \%$ and reaches up to $100 \%$ during the monsoon season in July-August and October-November. Temperature varies from $20-35^{\circ} \mathrm{C}$. Due to the typical climatic conditions and topographic characteristics of this area, P-VWS presents a remarkable diversity in vegetation and forest types. As per the classification by Chandrasekaran (1962), and, Champion and Seth (1968) west coast semi evergreen, southern moist mixed deciduous and southern subtropical savannahs are the major forest types of this area. Evergreen forest represents a climax vegetation type in P-VWS and small patches of this type of vegetation occur on the higher slopes of Vellanimala hill intermingled with semi-evergreen and deciduous patches especially near the vicinity of watercourses where suitable microclimate prevails. Semi-evergreen forests are usually found at relatively lower elevations and occur as a transition zone between moist deciduous and evergreen forests. The vegetation of moist deciduous forests is characteristic in that the trees of the upper canopy shed their leaves during the dry season from February to April. Xylia xylocarpa, Terminalia bellerica, Terminalia tomentosa, Garuga pinnata, Cinnamomum spp., Bridelia retusa, Grewia tiliaefolia and Haldina cordifolia are the common tree species. In the lower canopy, Ixora spp., Lantana camara and Clerodendrum spp. occur as undergrowth. A considerable portion of the forest area in this region has been converted to teak and eucalyptus plantations by the Forest Department. A variety of wild animals including tiger, elephant, gaur, sambar and bear, have been reported from this Sanctuary. Not many studies have been made on the insect fauna of this region except for a study on the insects associated with various tree species (Nair et al., 1986). The area was declared as a wildlife sanctuary in 1958.

\section{Materials And Methods}

Sampling of insects was done using a battery-operated Mathew's model light trap (Mathew \& Rahmathulla, 1995) at different locations in P-VWS. In addition to trap catches, collections were also made during daytime (0800 to $1300 \mathrm{hr})$ using hand nets. Insects thus collected were sorted and identified by comparison with material available in the Kerala Forest Research Institute collections, and referring to experts.

\section{Results AND Discussions}

Altogether 340 species of insects belonging to eight orders were collected during the survey, of which 321 species could be identified as given in Table 1. Of the various groups recorded, Lepidoptera and Coleoptera contained maximum number of taxa. Lepidoptera included 71 species of butterflies and 113 species of moths and Coleoptera 78 species. The other groups recorded were Hymenoptera (30 species), Hemiptera (15 species), Diptera (26 species), Orthoptera (1 species), Dictyoptera (4 species), and Isoptera (2 species).

The butterflies recorded in this study belong to seven families with Papilionidae and Nymphalidae containing maximum number of species followed by Pieridae and Satyridae. Six species of butterflies (Chilasa clytia, Appias lyncida, Appias libythea, Mycalesis anaxias, Hypolimnas misippus and Castalius rosimon) are undeer the protective schedules of the Indian Wildlife (Protection) Act; four species (Papilio buddha, Papilio polymnestor, Troides minos and Cirrochroa thais) are rather rare and restricted in distribution. Eight species of butterflies recorded in this study (Papilio dravidarum, Papilio hector, Hypolimnas misippus, Parantica nilgiriensis, Appias indra,

(C) Zoo Outreach Organisation; www.zoosprint.org Manuscript 1280; Received 02 November 2004; Finally accepted 06 July 2005; Date of publication 21 July 2005 


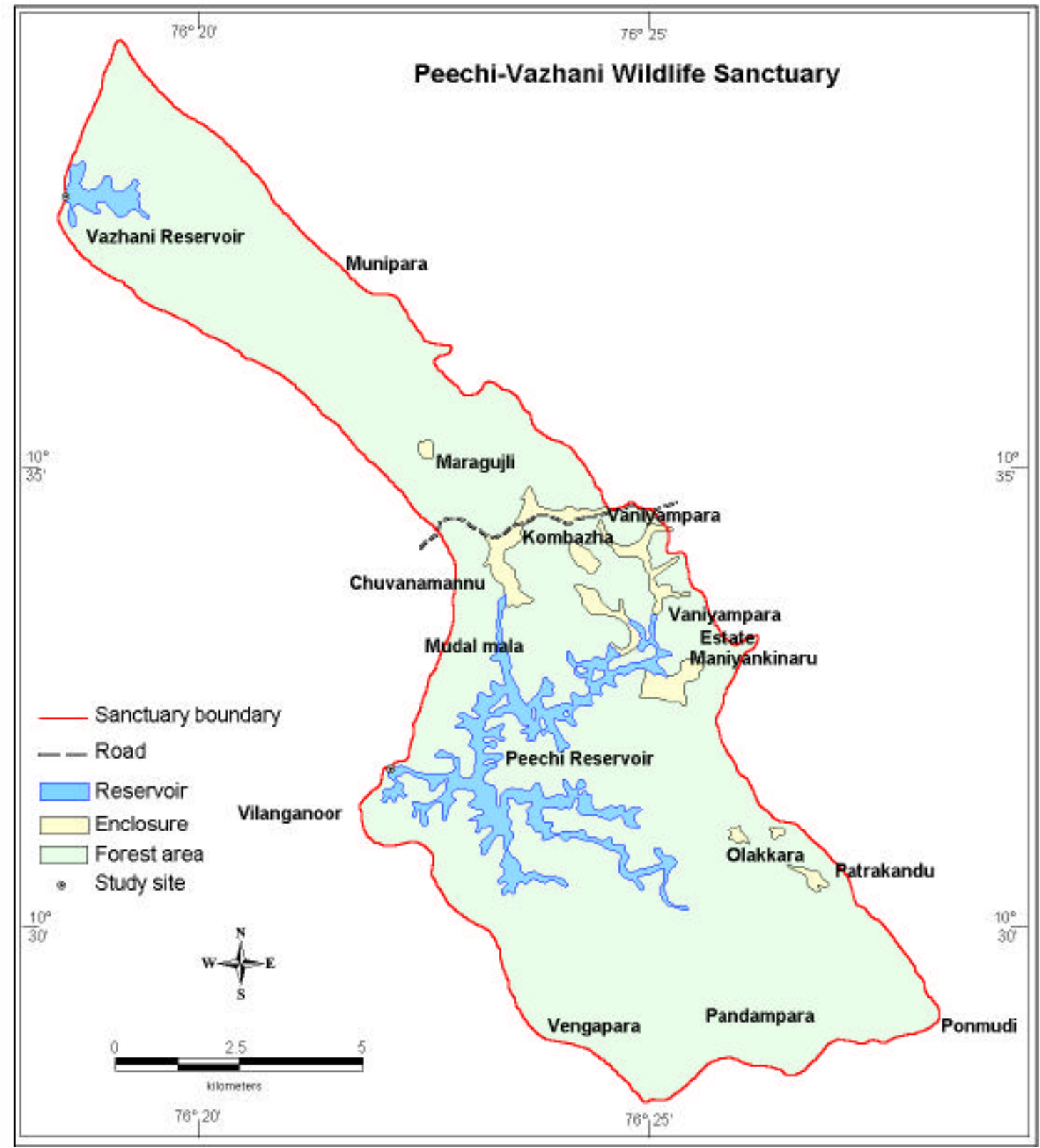

Figure 1. Map of the Peechi-Vazhani Wildlife Sanctuary, Thrissur district, Kerala.

Cepora nadina, Mycalesis igilia and Euchrysops cnejus) are found to be of high conservation status being either endemic or protected.

The moth fauna was very rich with the families Pyralidae and Noctuidae containing maximum number of species followed by Geometridae and Arctiidae. Information pertaining to the economic importance of a few species of moths are already available while that of the majority is still unknown. The moths of economic importance included Achaea janata (Noctuidae) attacking vegetable crops, the pyralids Cnaphalocrocis medinalis (attacking rice), Psara licarsicalis (attacking pulses), Glyphodes celsalis and Glyphodes bicolor (Pyraustidae) attacking various forest trees and Atteva fabricella
(Yponomeutidae) attacking Ailanthes triphysa.

The beetles included phytophagous, xylephagous, predatory and scavenger forms. The phytophagous beetles mostly belong to the family Chrysomelidae. This included Aulacophora cincta and Hoplasoma unicolor feeding on herbaceous flora. The families Cerambycidae, Bupersidae, Bostrychidae, Platypodidae and Curculionidae contain the xylephagous forms. The scavenger beetles included the scarabaeids Copris sp., Maladera sp. (attacking foliage of Mangium), Oryctes rhinoceros (attacking Palm) and Popillia sp. (attacking petals of rose).

With regard to Hymenoptera, several species of social wasps 
Table 1. List of insects recorded from Peechi-Vazhani Wildlife Sanctuary

\begin{tabular}{|c|c|c|c|}
\hline Order / Family & Remarks & Order / Family & Remarks \\
\hline Lepidoptera & & Potanthus pava pava Fruhstorfer & Common \\
\hline Rhopalocera & & Pelopidas mathias $\mathrm{Fb}$. & Fairly common \\
\hline Papilionidae & & Tagiades litigiosa Moschler & Fairly common \\
\hline Chilasa clytia Lin. & Rare & Taractrocera ceramas (Hewit.) & Rare \\
\hline Graphium sarpedon Felder & Common & Telicota acigias Lin. & Fairly common \\
\hline Graphium agamemnon agamemnon Lin. & Common & Lvcaenidae & \\
\hline Graphium doson doson Felder & Common & $\begin{array}{l}\text { Lycaenidae } \\
\text { Arhopala centaurus Moore }\end{array}$ & Common \\
\hline Papilio polytes Cramer & Common & $\begin{array}{l}\text { Arnopala centaurus IVoore } \\
\text { Castalius rosimon (Fb.) }\end{array}$ & $\begin{array}{l}\text { Common } \\
\text { Rare Schedule II }\end{array}$ \\
\hline Papilio helenus Lin. & Common & Curetis ?thetis Drury & Rare \\
\hline Papilio polymnestor parinda Moore & Common & Euchrysops cnejus (Fb.) & Common \\
\hline Papilio buddha Westwood & $\begin{array}{l}\text { Rare, endemic to Western Ghats, } \\
\text { Schedule II (IWP Act, 1972) }\end{array}$ & Jamides alecto (Felder) & Rare \\
\hline \multirow[t]{2}{*}{ Papilio dravidarum Wood-Mason } & \multirow{2}{*}{$\begin{array}{l}\text { Rare, endemic to Western } \\
\text { Ghats }\end{array}$} & Thalicada nyseus (Guerin.) & \multirow[t]{2}{*}{ Fairly common } \\
\hline & & Heterocera & \\
\hline Papilio demoleus demoleus Lin. & Common & Oecophoridae & \\
\hline Pachliopta aristolochiae Lin. & Common & Aeolonthes dicraea Meyrick & \\
\hline Pachliopta hector Lin. & Common & Tonica sp. & Borer in Bombax \\
\hline Troides minos Cram. & Endemic to Western Ghats & Yponomeutidae & \\
\hline Nymphalidae & & Atteva fabriciella Swed. & Pest of Ailanthus \\
\hline Argynnis hyperbius (Johannsen) & Common & & \\
\hline Ariadne merione Cramer & Uncommon & Gelechiidae & \\
\hline Cyrestis thyodamas Kollar & Rare & Dichomeris sp. & \\
\hline Cirrochroa thais thais $\mathrm{Fb}$. & Rare & Cossidae & \\
\hline Cupha erymanthis Drury & Common & Alceterogystia (=Cossus) cadambae (Moore) & Teak carpenter worm \\
\hline Hypolimnas bolina Lin. & Common & & \\
\hline Hypolimnas misippus Lin. & Common, Schedules I \& II & Psychidae & \\
\hline Neptis hylas varmona Moore & Very common & Metisa plana WIk. & \\
\hline Neptis perius Fruhstorfer & Rare & Eumeta crameri West. & Tea, Ailanthus triphysa, \\
\hline Phalanta phalantha Drury & Common & & Acacia arabica \\
\hline Junonia atlites Lin. & Common & Brachycyttarus Sp. & \\
\hline Junonia hierta Fb. & Very common & Pteroma plagiophleps Hamp. & Pest of Albizia spp., Tamarind \\
\hline Junonia iphita Fruhstorfer & Rare & Thyridopteryx sp. & \\
\hline Junonia lemonias Fruhstorfer & Common & Noctuidae & \\
\hline Kaniska canace Moore & Rare & Achaea janata $\mathrm{Fb}$. & Pest of vegetables \\
\hline Moduza procris Cram. & Common & Achaea flava (Fb.) & - \\
\hline Danaidae & & Carea endophaea Hamp. & Pest of syzygium \\
\hline Danaus genutia genutia Cramer & Common & Carea subtilis WIk. & Pest of syzygium \\
\hline Danaus chrysippus (Lin.) & Common & Chasmina rejecta $\mathrm{Fb}$. & - \\
\hline Euploea core core Cramer & Very common & Heliothis sp. & Crop pest \\
\hline Parantica aglea Stoll & Fairly common & Mocis frugalis $\mathrm{Fb}$. & Pest of fruits \\
\hline Tirumala limniace leopardus Butler & Common & Mythimna curvilinea Hamp. & - \\
\hline Tirumala septentrionis dravidarum & Rare & Othreis materna Lin. & Fruit moth \\
\hline Fruhstorfer & & Othreis fullonica Lin. & Fruit moth \\
\hline Parantica nilgiriensis Moore & Rare & Othreis ancilla Cram. & Fruit moth \\
\hline & & Polytela gloriosae $\mathrm{Fb}$. & Pest of Lilly \\
\hline Pieridae & & Prodenia litura $\mathrm{Fb}$. & Crop pest \\
\hline Appias indra Moore & Common & Spiredonia retorta Cram. & $\begin{array}{l}- \\
0\end{array}$ \\
\hline Appias lyncida (Cramer) & Uncommon & Spodoptera litura (Fb.) & Crop pest \\
\hline Appias libythea $\mathrm{Fb}$. & Uncommon & Spodoptera mauritia Boisduval & Crop pest \\
\hline Catopsilia florella (Fb.) & Fairly common & Earias flavida sulphuraria Moore & Garuga pinnata \\
\hline Catopsilia pomona $\mathrm{Fb}$. & Very common & Plecoptera reflexa & Pest of Dalbergia sissoo \\
\hline Catopsilia pyranthe (Lin.) & Very common & Tiracola plagiata WIk. & - \\
\hline Cepora nadina Moore & Rare & Oglasa sp. & - \\
\hline Eurema blanda Boisd. & Common & & \\
\hline Eurema brigitta Stoll & Common & $\begin{array}{l}\text { Hyblaeidae } \\
\text { Hyblaea puera Cram. }\end{array}$ & \\
\hline Eurema hecabe Lin. & Common & Hyblaea puera Cram. & Teak defoliator \\
\hline $\begin{array}{l}\text { Delias eucharis Drury } \\
\text { Eurema laeta Boisd. }\end{array}$ & Common & Lymantriidae & \\
\hline Eurema laeta Boisd. & Rare & Dasychira mendosa $\mathrm{Hb}$. & Crop pest \\
\hline Eurema lacteola Dist. & Rare & Euproctis fraterna Moore & Bombax sp. \\
\hline Satyridae & & Eupterotidae & \\
\hline Elymnias caudata Butler & Common & Eupterote flavida Moore & - \\
\hline $\begin{array}{l}\text { Melanitis leda Lin. } \\
\text { Mycalesis anaxias Hewitson }\end{array}$ & Very common & Aratiidar & \\
\hline $\begin{array}{l}\text { Mycalesis anaxias Hewitson } \\
\text { Ypthima sp. }\end{array}$ & $\begin{array}{l}\text { Uncommon, Schedule II } \\
\text { Fairly common }\end{array}$ & $\begin{array}{l}\text { ArctIIdae } \\
\text { Amata extensa Wlk }\end{array}$ & _ \\
\hline Melanitis phedima varaha Moore & Rare & Amata argus Koll. & - \\
\hline Ypthima ceylonica Hewit. & Fairly common & Amata astrea Drury & Pest of legumes \\
\hline Mycalesis patnia Moore & Rare & Amata cribraria Clerck & Pest of legumes \\
\hline Mycalesis igilia $\mathrm{Fb}$. & Rare & Asura conferta WIk. & Pest of mosses \\
\hline Lethe europa $\mathrm{Fb}$. & Rare & Creatonotus gangis Lin. & Pest of lilly \\
\hline & & Diacrisia obliqua Wlk. & Polyphagous pest \\
\hline Hesperidae & & Eligma narcissus Cram. & Pest of Ailanthus triphysa \\
\hline $\begin{array}{l}\text { Celaenorrhinus ambareesa (Moore) } \\
\text { Celaenorrhinus leucocera (Kollar) }\end{array}$ & Common & Estigmene perotetti & Pest of bamboo \\
\hline Celaenorrhinus leucocera (Kollar) & Common & Pericallia ricini $\mathrm{Fb}$. & Pest of castor \\
\hline
\end{tabular}




\begin{tabular}{ll}
\hline Order / Family & Remarks \\
\hline Geometridae & \\
Abraxas sp. nr. latizonata Hamp. & Feeds of foliage of forest trees \\
Boarmia infixariaWlk. & Pest of Xylia xylocarpa \\
Buzura? suppressaria Wlk. & Feeds of foliage of forest trees \\
Cleora sp. prob. alienaria WIk. & Feeds of foliage of forest trees \\
Epiplema quadricaudata WIk. & Pest of Haldina cordifolia \\
Epiplema fulvilinea Hamp. & Pest of Gmelina arborea \\
Eumelea rosalia Cram. & - \\
Eumelea sp. & - \\
Hypochrosis sp.? abstractaria Wlk. & Pest of Xylia xylocarpa \\
Hypomecis sp. & Pest of Xylia xylocarpa \\
Hyposidra talaca Wlk. & Feeds of foliage of forest trees \\
Rhesala moestalis WIk. & - \\
Sabaria euchroes Prout & - \\
Semiothisa quadraria Moore & Feeds of foliage of forest trees \\
Thalassodes ?opalina Warr. & Bombax sp. \\
\end{tabular}

Pterophoridae

Diacrotricha leucomochla Fletcher

Xyroptlia tectonica Meyrick

\section{Pyraustidae}

Agathodes ostentalis Hubn.

Agrotera basinotata Hamp.

Botyodes asialis Guen.

Bocchoris inspersalis Zell.

Bradina admixtalis WIk.

Cirrhochrista fumipalpis Feld.

Diaphania (Glyphodes) glauculalis Guen. Pest of Tabernaemontana

Dichocrocis punctiferalis Guen.

Eurrhyparodes tricoloralis Zell.

Filodes fulvidorsalis Hubn.

Glyphodes celsalis WIk.

Glyphodes bicolor Swains.

Glyphodes laticostalis Guen.

Glyphodes indica Saund.

Glyphodes itysalis WIk.

Glyphodes marginata Hamp.

Isocentris filalis Guen.

Lamprosemasp.

Marasmia trapezalis Guen.

Nacoleia diemenalis Guen.

Nymphula fluctuosalis Zell.

Nymphula foedalis Guen.

Protrigonia zizanialis Swinh.

Parotis (Glyphodes) vertumnalis Guen.

Psara bipunctalis $\mathrm{Fb}$.

Pycnarmon caberalis Guen.

Pygospila tyres Cram.

Sylepta derogata $\mathrm{Fb}$.

Syngamia abruptalis WIk.

Syngamia latimarginalis WIk.

Syngamia sp.

Terastia egialealis WIk.

Lygropia orbinusalis WIk.

Eutectona machaeralis WIk.

Pyralidae

Antigastra catalunalis Swinh.

Cnephalocrocis medinalis Guen.

Deba survectalis WIk.

Lamida moncusalis WIk.

Macalla ?eumictalis Hamp.

Macalla nubilalis Hamp.

Crambidae

Ancylolomia chrysographella Kollar

Charltona consociella WIk.

Chilo polychrysa Meyrick

Chilo partellus Swinh.

\section{Phycitidae}

Etiella zinckenella Treit.

Nephoteryx atrisquamella Hamp.

Pest of Gmelina arborea

Crop pest

Pest of Graminae

Borer in casto

Feeds on lachrymal secretion of animals

Pest of forest trees

Pest of forest trees

Pest of forest trees

Pest of cucumber

Pest of forest trees

Pest of pulses

Pest of grasses

Pest of pulses

Pest of Graminae

Pest of Graminae

Pest of drumstick

Pest of Haldina cordifolia,

Alstonia scholaris

Pest of pulses

Pest of coleus

Pest of malvaceaus plants

Pest of Ocimum

Pest of Erythrina

Grewia tiliaefolia

Tectona grandis

Pest of gingelli

Pest of rice / graminaceous plants

Cassia fistula

Pest of mango tree, Terminalia bellirica

Pest of mango, Garuga pinnata

Pest of rice, jowar

Pest of Graminae

Pest of rice

Pest of Graminae

Pod borer of pulses crops

\begin{tabular}{ll}
\hline Order / Family & Remarks \\
\hline Assara albicostalis Wlk. & Pest of Garuga pinnata \\
Ectomyelois ceratoniae (Zell.) & \\
Phycita spp.+ &
\end{tabular}

Galleriidae

Galleria mellonellaLin. Pest of bees

Sphingidae

Acherontia lachesis $\mathrm{Fb}$ Crop pest

Acherontia sp. Crop pest

Herse convolvuli Lin. Crop pest

Oxyambulyx sp.

Theretra sp.

Oxyambulyx subocellata Feld.

Crop Pest

Saturnidae

Attacus atlas Lin.

Loepa sikkima Moore

\section{Cosmopterygidae}

Limnoecia sp.? peronodes Meyrick

Pterophoridea

Xyroptila tectonica+

Melarbelidae

Arbela tetraonis+

Totricidae

Adoxophyes moderatana WIk.

Garuga pinnata

Thyrididae

Striglina scitaria Wlk.+

\section{Coleoptera:}

Lucanidae

Odontolabis cuvera Hope? Host of decaying wood

Odontolabis sp.

Coccinellidae

Epilachna septima Dieke

Epilachna vigintioctopunctata Fb.

Epilachna sp.

Coelophorasp.

Coccinella septempunctata Lin.

Catana parcestosa (Sicard)

\section{Scarabaeidae}

Adoretus bicaudatus Arrow

Adoretus coronatus Burm.

Anomala ruficapilla Burmeister

Anomala sp.

Copris sp.

Heliocopris dominus Bates

Heterorrhina sp.

Holotrichia rufoflava Brenske

Maladera sp.

Mimela sp.

Mimela xanthorrhina Hope

Popillia complanata Newm.

Adoretus sp.

Atlas moth

Pest of vegetable crops Pest of Gmelina arborea

Lagerstroemia sp.

Garuga pinnata

Adult feeds on foliage of forest plants

Adult feeds on foliage of forest plants

Dung rolling beetle

Dung rolling beetle

Feed on dung

Grubs feed on roots causing

seedling mortality

Adults feed on foliage of mangium Adults feed on foliage of mangium Adults feed on foliage of mangium Adults feed on petals of rose Grubs feed on roots causing seedling mortality

\section{Elateridae}

Agriotes sp.

Agrypnus holosericeus Candeze

Campsosternus sp.

Garuga pinnata

Chrysomelidae

Apophylea sericea (Fb.)

Apophylea cincta (Fb.)

Aulacophora unicolor Illig.

Basilepta fulvicornis Jac.

Hoplasoma unicolor Illig.

Monolepta longitarsis Jac.

Lema yerburyi Jac.

Lilioceris ? laosensis Jac.

Heptis sp., Garuga pinnata

Feeds on foliage of Pumkin

Feeds on foliage of Pumkin

Feeds on foliage of herbs

Feeds on foliage of herbs

Feeds on foliage of herbs

Feeds on foliage of herbs

Feeds on foliage of herbs

Demarchus pubipennis Jac.

Feeds on foliage of herbs 


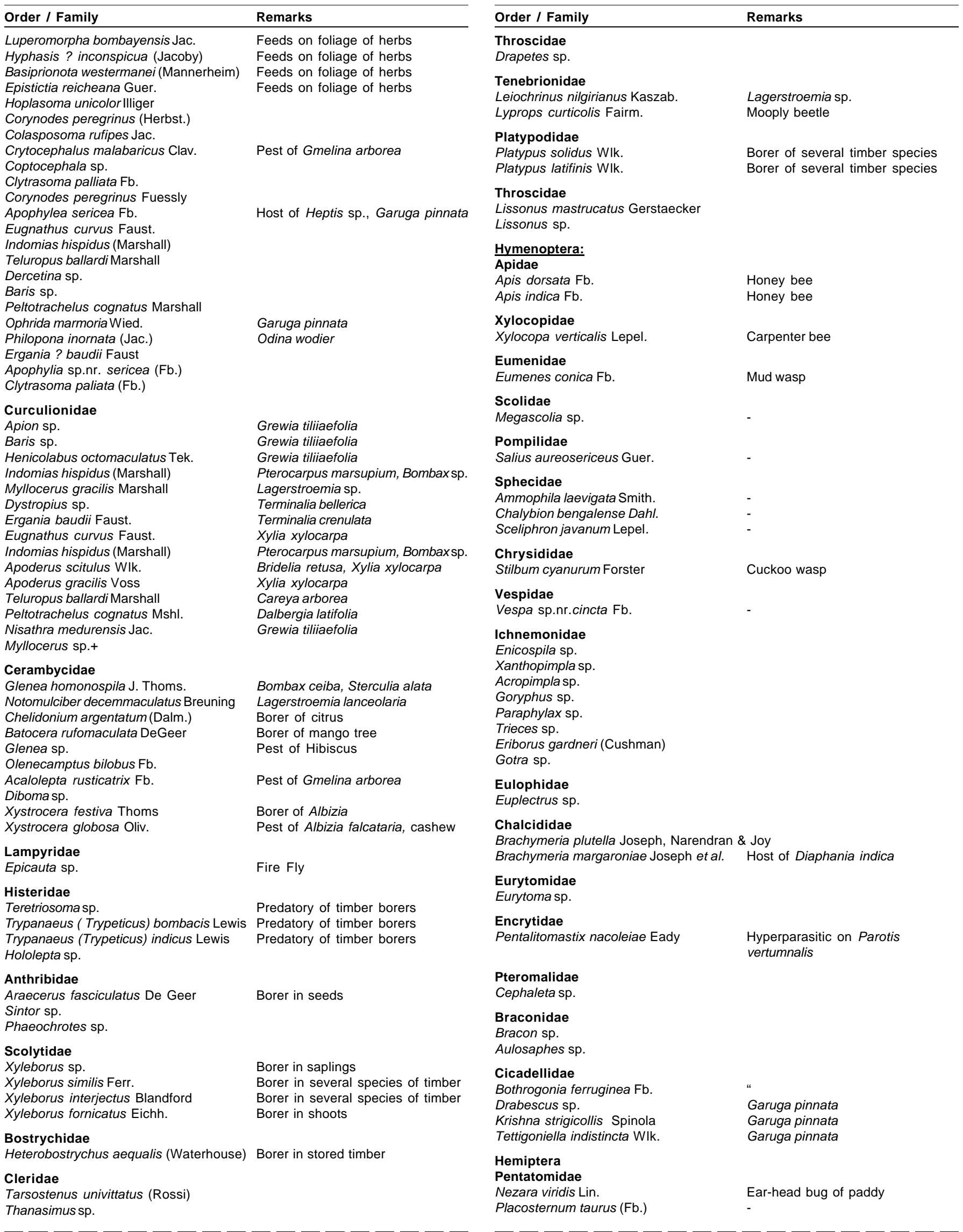




\begin{tabular}{|c|c|}
\hline Order / Family & Remarks \\
\hline $\begin{array}{l}\text { Dysdercus cingulatus } \mathrm{Fb} . \\
\text { Serinatha augur } \mathrm{Fb} .\end{array}$ & Cotton bug \\
\hline $\begin{array}{l}\text { Miridae } \\
\text { Helopeltis antonii Signoret }\end{array}$ & $\begin{array}{l}\text { Tea mosquito, Pest of tea and } \\
\text { cashew }\end{array}$ \\
\hline $\begin{array}{l}\text { Tingitidae } \\
\text { Tingis beesoni Drake }\end{array}$ & Pest of Gmelia arborea \\
\hline $\begin{array}{l}\text { Coreidae } \\
\text { Acanthocoreis strucornis (Scott.) }\end{array}$ & Pest of Albizia \\
\hline $\begin{array}{l}\text { Ricaniidae } \\
\text { Ricania speculum Walker } \\
\text { Ricania marginalis ?Auther } \\
\text { Ricania sp. }\end{array}$ & \\
\hline $\begin{array}{l}\text { Flattidae } \\
\text { Flatta ferrugata } \mathrm{Fb} .\end{array}$ & \\
\hline $\begin{array}{l}\text { Cercopidae } \\
\text { Phymatostetha deschampsi Leth. } \\
\text { Poophilus sp.+ }\end{array}$ & Sap sucking on Dalbergia latifolia, \\
\hline $\begin{array}{l}\text { Psyllidae } \\
\text { Phacopteron lentiginosum Buckton }\end{array}$ & Garuga pinnata \\
\hline $\begin{array}{l}\text { Membracidae } \\
\text { Tricentrus sp. } \\
\text { Garagara sp. } \\
\text { Centrotypus sp. }\end{array}$ & \\
\hline $\begin{array}{l}\text { Coccidae } \\
\text { Ceroplastes sp. }\end{array}$ & \\
\hline $\begin{array}{l}\text { Plataspidae } \\
\text { Coptosoma variegata+ }\end{array}$ & \\
\hline $\begin{array}{l}\text { Diptera } \\
\text { Cecidomyidae } \\
\text { Asphondylia sp. } \\
\text { Asphondylia tectonae Mani }\end{array}$ & $\begin{array}{l}\text { Teak gall insects } \\
\text { Tectona grandis }\end{array}$ \\
\hline $\begin{array}{l}\text { Tachinidae } \\
\text { Palexorista sp. } \\
\text { Blepharipa sp. } \\
\text { Carcelina sp. } \\
\text { Thecocarcelia sp. } \\
\text { Thelairodrino sp. }\end{array}$ & \\
\hline $\begin{array}{l}\text { Tephritidae } \\
\text { Dacus (Bactrocera) sp.nr. tuberculatus+ }\end{array}$ & \\
\hline Muscidae - 10 spp. & \\
\hline Tabanidae - 2 spp. & \\
\hline Syrphidae - 2 spp. & \\
\hline Culicidae - 5 spp. & \\
\hline $\begin{array}{l}\text { Orthoptera } \\
\text { Acrididae } \\
\text { Catantops sp. }\end{array}$ & - \\
\hline$\frac{\text { Dictyoptera }}{\text { Mantidae }}$ & \\
\hline $\begin{array}{l}\text { Deroplatys desicata West } \\
\text { Statialia maculata (Thunberg) } \\
\text { Humbertiella indica Sauss. }\end{array}$ & $\begin{array}{l}\text { Preying mantis } \\
\text { Preying mantis } \\
\text { Preying mantis }\end{array}$ \\
\hline $\begin{array}{l}\text { Phasmidae } \\
\text { Phasmida sp. }\end{array}$ & Stick insect \\
\hline $\begin{array}{l}\text { Isoptera } \\
\text { Termitidae } \\
\text { Pericapritermes vythirii Verma } \\
\text { Odontotermes obesus Ramb. }\end{array}$ & \\
\hline
\end{tabular}

belonging to the families Eumenidae, Eulophidae, Sphecidae; parasitic wasps belonging to the families Ichneumonidae and Braconidae and bees belonging to the families Apidae and Xylocopidae were recorded. The bugs contained several species of economic significance such as the earhead bug of paddy Nezara viridis, the cotton bug Dysdercus cingulatus (Pentatomidae), the tea mosquito Helopeltis antonii (attacking tea and cashew), the lace bug Tingis beesoni (attacking Gmelina arborea) and the albizia bug Acanthocoreis strucornis. Of the 26 species of dipteran flies collected, only six could be identified. With regard to Isoptera, two species, viz., Pericapritermes vythirii and Odontotermes obesus were identified. In addition to these, preying mantis (Mantidae), stick insects (Phasmidae) and grasshoppers (Orthoptera) were also recorded. Nair et al. (1986) had earlier reported 85 species of insects associated with 20 tree species in the Peechi-Vazhani Wildlife Sanctuary of which except for eight species, all have been recorded in this study (Table 1).

\section{REFERENCES}

Champion, H.G. and S.K. Seth (1968). A Revised Survey of the Forest Types of India. Govt of India Press, Nasik, 323pp.

Chandrasekharan, C. (1962). Forest types of Kerala State. Indian Forestry 88: 660-847.

Mathew, G. and V.K. Rahmathulla (1995). Biodiversity in the Western Ghats - a study with reference to moths in the Silent Valley National Park, India. Entomon 20(2): 25-33.

Nair, K.S.S., G. Mathew, K. Mohanadas and K.S.S. Menon (1986). A study of insects pest incidence in natural forests. KFRI Research Report No. 44, 28pp.

\section{ACKNOWLEDGEMENTS}

We thank Dr. T.C Narendran, Retd. Professor and Head, Department of Entomology, Calicut University, for kindly identifying several species of insects collected in this study.

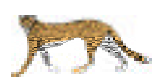

